\title{
Discrete Integral Sliding Mode Control in Visual Object Tracking Using Differential Kinematics
}

\author{
Luis Enrique González Jiménez, Alexander Loukianov, \\ and Eduardo Bayro-Corrochano \\ CINVESTAV, Department of Automatic Control, Unidad Guadalajara, \\ Av. Científica 1145, 45015 Zapopan, Jalisco, México \\ \{lgonzale, louk, edb\} @gdl.cinvestav.mx
}

\begin{abstract}
A Discrete Integral Sliding Mode algorithm is proposed to control a Stereo Vision System (SVS) and perform Visual Object Tracking. The kinematical model of the structure is obtained using Geometric Algebra (GA). The localizing part was done in a real SVS in order to obtain the reference for orientation vector and the application for a Pan Tilt Unit is presented. The algorithm presents a good and robust performance.
\end{abstract}

Keywords: Integral Sliding Mode Control, Visual Tracking, Geometric Algebra.

\section{Introduction}

Consider a stereo vision system (SVS) that is mounted on a mechanical device which defines the system's orientation related to a defined base frame. The global task can be divided in two parts: first the system must recognize the target in the scene and extract a vector that will characterize the object's position; then the system must re-define its kinematical structure, so its orientation can be aligned with the target's position. Using a stereo system provides the advantage of depth information, this is fundamental in tasks like grasping and manipulating objects, in addition to visual tracking.

\subsection{Target Localization}

Model-based algorithms use a pre-obtained model of the object, usually composed of lines. From this model, points of interest are projected on the image plane, and when the correspondences of these points are located and the image, the target is located as well [1]. Global Appearance-based methods segment a region from the image containing the object. Among the algorithms used for feature detection are the Harris corner detector and SIFT [2]. Histogram-based methods use histograms obtained from the target and it is compared with a reference image in the scene. The histogram uses usually color [3] or spatial information.

\subsection{Kinematical Control}

Several algorithms have been used to control robotic devices such as the classic PID controller, Adaptive Control [4], Neural Control [5] and Sliding Mode Control (SMC) 
[6]. Among these methods SMC is one of the most effective approaches due to its robustness to matched perturbations, model uncertainties, and it demands a low computational cost. On the other hand, the Integral Sliding Mode Control (ISMC) [7] can guarantee the robustness of the closed-loop system throughout the entire response starting from the initial time instance, and permits to reduce the controller gains in comparison with standard SMC. However, due to a finite sampling rate, continuoustime sliding mode control could be inappropriate for a discrete-time system. Hence, a re-work in the sliding-mode control (SMC) strategy for sampled-data systems was necessary [9]. The equivalent control is used in this work to design a chattering-free discrete ISM controller (DISMC) for a SVS, and a performance of this controller is demonstrated.

\section{Problem Formulation}

A general scheme for a SVS is presented in Figure 1, where $T_{p}$ is the target's point, $A$ is the orientation vector for the SVS, $O_{b}$ is the origin of the base frame and $O_{c}$ is the origin of the camera frame conveniently attached to the last link of the kinematical device.

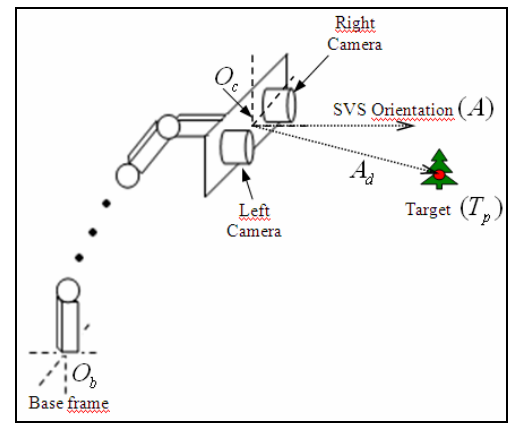

Fig. 1. Stereo Vision System and target

The kinematical model of this structure can be defined as [8]

$$
A=f(\theta)
$$

where $\theta=\left[\theta_{1}, \ldots, \theta_{n}\right]^{T}$ is the angles vector of the system, $n$ is the number of joints in the kinematical structure and the vector $f(\theta)$ is defined by the direct kinematics of the system.

\subsection{Kinematical Model with GA}

The kinematical model for a serial manipulator can be obtained using a Geometric Algebra approach [10], which has the advantage of being a simple procedure. In this work geometric algebra $G_{3,0,0}$ with the orthonormal base $\left\{e_{1}, e_{2}, e_{3}\right\}$ is used. 
First we need to define the rotor (geometric entity that defines rotations) for each joint of the system as

$$
R_{i}=e^{-\frac{1}{2} L_{i} \theta_{i}}
$$

where $L_{i}$ is the axis of rotation for the $i^{\text {th }}$ joint given by

$$
L_{1}=L_{1,0} \text { and } L_{i}=R_{i-1} \ldots R_{1} L_{i, 0} R_{1} \ldots R_{i-1} \text { for } i=2, \ldots, n .
$$

Then, the actual orientation of the last link $A$ is obtained of the form

$$
A=R_{n} \ldots R_{1} A_{0} \tilde{R}_{1} \ldots \tilde{R}_{n}
$$

where $n$ is the number of joints (degrees of freedom) of the system, $A_{0}$ the initial orientation $\left(\theta_{1}=\ldots=\theta_{n}=0\right)$ and $\tilde{R}_{i}$ is the reverse vector for $i^{\text {th }}$ joint given by $\tilde{R}_{i}=e^{\frac{1}{2} L_{i} \theta_{i}}$. Differentiation of equation (2) yields

$$
\dot{A}=J_{A} \dot{\theta}
$$

where $J_{A}$ is the Jacobian matrix. This equation defines the Differential Kinematics of the system.

\subsection{Discrete State-Space Model}

Defining the reference orientation $A_{d}$ as the vector conformed by the target's point and the origin of the camera frame, a control error variable can be obtained as follows:

$$
e_{A}=A-A_{d}
$$

Then, using (4) and (5), and adding a disturbance term $\gamma(t)$, due to uncertainties in the model, we obtain:

$$
\dot{e}_{A}=J_{A} \dot{\theta}-\dot{A}_{d}+\gamma(t) .
$$

Assuming the terms $\dot{A}_{d}$ and $\gamma(t)$ are unknown, representing them in a perturbation vector $d(\theta, t)$ and considering $\dot{\theta}$ as the control vector $U$, the equation (6) can be reformulated as

$$
\dot{e}_{A}=J_{A} U-d(\theta, t) .
$$

We assume that function $d(\theta, t)$ is smooth and bounded by known positive scalar function

$$
\|d(\theta, t)\|<\beta_{2}(\theta, t) .
$$

Using Euler's discretization, and defining the state-space variables as follows

$$
\begin{aligned}
& x_{1, k}=\theta_{k} \\
& x_{2, k}=A_{k}
\end{aligned}
$$


we can obtain the discrete state-space model for the system as

$$
\begin{aligned}
& x_{1, k+1}=x_{1, k}+T u_{k} \\
& x_{2, k+1}=x_{2, k}+T J_{A, k} u_{k}
\end{aligned}
$$

where $x_{i, k}=x_{i}(k T)$ for $i=\{1,2\}$, and $T$ is the sample time. Defining the output of the system as $y_{k}=x_{2, k}$ and the discrete error variable for the system (9) as

$$
e_{A, k}=y_{k}-A_{d, k}
$$

where $A_{d, k}$ is the discrete version of the reference vector, then the dynamics for the error system can be obtained using (9) and (10) as

$$
e_{A, k+1}=x_{2, k}+T J_{A, k} u_{k}-d_{k}
$$

with $d_{k}$ as the discretization of perturbation vector.

Now, the problem considered here is to design a Discrete Integral Sliding Mode Controller (DISMC) that ensures visual object tracking in despite of external disturbance $d_{k}$.

\section{DISM Controller Design}

Consider the sliding function $s_{k}$ as

$$
s_{k}=e_{A, k}+z_{k}
$$

where $z_{k}$ is the integral variable which is given by the following equation

$$
z_{k+1}=z_{k}-T G e_{A, k}
$$

electing $z_{0}=-e_{0}$ to ensure sliding mode occurrence on the sliding manifold $s_{k}=0$ from initial instance and $G$ is a design parameter.

From (11) and (12), the projection motion of the system on the subspace $s_{k}$ can be obtained of the form

$$
s_{k+1}=x_{2, k}+T J_{A, k} u_{k}-d_{k}+z_{k+1}
$$

We define the control as

$$
u_{k}=-\frac{J_{A, k}^{+}}{T}\left(x_{2, k}-\hat{d}_{k}+z_{k}-T G e_{k}+K e_{k}\right)
$$

where $J_{A, k}^{+}$is the pseudo-inverse of $J_{A, k}, \hat{d}_{k}$ is the estimation of perturbation term and $K$ is a constant which will be defined later. From (6) and (7) we know that $d_{k}$ is the derivative of reference vector $A_{d}$, so we can choose its estimation as

$$
\hat{d}_{k}=A_{d, k}-A_{d, k-1}
$$

Then, the closed loop system (11),(14) and (15) becomes 


$$
\begin{aligned}
e_{k+1} & =(T G-K) e_{k}-z_{k}+\varphi_{k} \\
s_{k+1} & =\varphi_{k}-K e_{A, k}
\end{aligned}
$$

where $\varphi_{k}=\hat{d}_{k}-d_{k}$, and we can formulate a theorem as follows:

Theorem 1. If the assumption (8) holds, the control law

$$
u_{k}=-\frac{J_{A, k}^{+}}{T}\left(x_{2, k}-\hat{d}_{k}+z_{k}-T G e_{k}+K e_{k}\right)
$$

is constructed, the inequality

$$
\frac{2}{T-2}<G<0
$$

holds and

$$
K=(T-1) G
$$

then a solution of the error dynamics (11) converges asymptotically to a vicinity of zero, and this vicinity is bounded by $\varphi_{k}-\varphi_{k-1}$.

The proof of the Theorem 1 can, unfortunately, not be included due to its length.

Thus, the control objective is fulfilled and the SVS with the proposed discrete controller performs tracking of the target.

\section{Application for a Pan-Tilt Unit}

The simulations results of this work were obtained applying the designed controller to a Pant-Tilt Unit (PTU). The base frame is defined by the unit vectors $\left\{e_{1}, e_{2}, e_{3}\right\}$.The angles vector is defined as $\theta=\left[\theta_{1}, \theta_{2}\right]^{T}$. For simplicity is assumed that the orientation of the second link $A$ is identical to the orientation of the principal axis of right camera, so there is no need of Hand-Eye calibration. The kinematical model for the PTU was obtained as follows. The axes of rotation of the PTU are given by $L_{1,0}=e_{3}$ and $L_{2,0}=e_{1}$, the actual orientation $A$ and the Jacobian matrix $J_{A}$ are calculated as

$$
A=\left[\begin{array}{c}
y_{1} y_{2} \\
-c_{1} y_{2} \\
c_{2}
\end{array}\right] \text { and } J_{A}=\left[\begin{array}{cc}
y_{2} c_{1} & y_{1} c_{2} \\
y_{1} y_{2} & -c_{1} c_{2} \\
0 & -y_{2}
\end{array}\right] \text {. }
$$

where $y_{i}=\sin \left(\theta_{i}\right)$ and $c_{i}=\cos \left(\theta_{i}\right), i=1,2$.

In order to obtain a real reference vector $A_{d, k}$, a color segmentation of a moving target in a SVS was developed. The SVS is composed of two Flea ${ }^{\circledR}$ cameras from Point Grey Research Inc. mounted on a metal bar as depicted in Figure 1. The segmentation was based on HSV color space (Hue-Saturation-Value). First, a calibration process for the SVS is realized. Then, the HSV parameters from the target are obtained and compared with the HSV values from the images, resulting in the segmentation of the target in the images. 

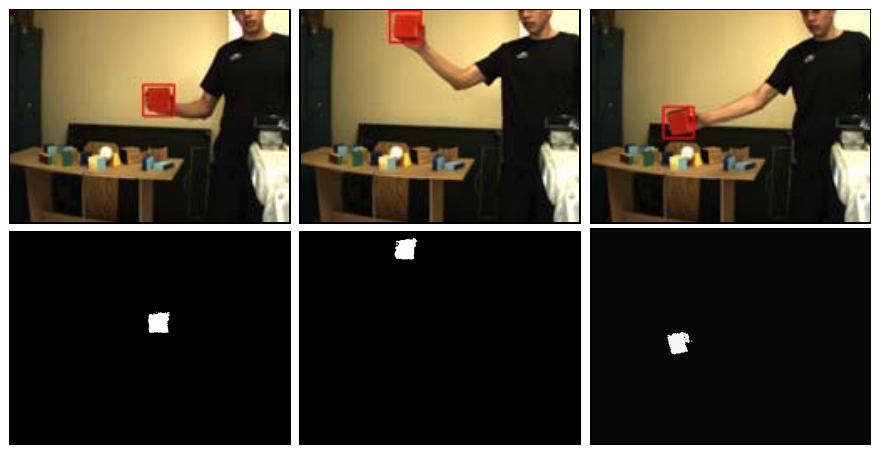

Fig. 2. Color Segmentation (Right Camera)

From segmentation the target is located with 2D coordinates for each image; then the $3 \mathrm{D}$ vector $V_{s}$, defined from the center of the right camera to the target $T_{p}$, is obtained. Figure 3 shows samples of the segmentation process (right column) and the corresponding locations in the image (left column) for the right camera. In total, 45 pairs of images were captured with a sample time of $T=220$ miliseconds. The control parameter for DISMC algorithm is $G=-0.5$ A parallel discrete PID algorithm, defined by the following equation

$$
u_{k}=u_{k-1}-J_{A, k}^{+}\left[K_{p} t_{s} e_{k}+\frac{K_{i}}{t_{s}}\left(e_{k}-2 e_{k-1}+e_{k-2}\right)+K_{d}\left(e_{k}-e_{k-1}\right)\right]
$$

was applied in simulation and the control gains used were

$$
K_{p}=15, K_{i}=0.5, K_{d}=1 .
$$

The disturbance term $\gamma_{k}$ and initial conditions used in both simulations were

$$
\gamma_{k}=\left[\begin{array}{c}
0.5 \\
0.2+0.3 \sin (\pi k T) \\
-3+0.2 \cos (k T)
\end{array}\right], \theta_{0}=\left[\begin{array}{c}
\pi / 2 \\
\pi / 3
\end{array}\right],
$$

and the sample time 220 milliseconds.

\section{Simulation Results}

Figure 3 shows the three components of the orientation vector and their references for DISMC and PID algorithms, respectively. It can be appreciated that the goal of control is fulfilled in both controllers, since the objectives are accomplished. However, the convergence in DISCM is smoother than PID, and less oscillatory. The error variables converge to a vicinity of zero in less time (settling time) for DISMC than for 

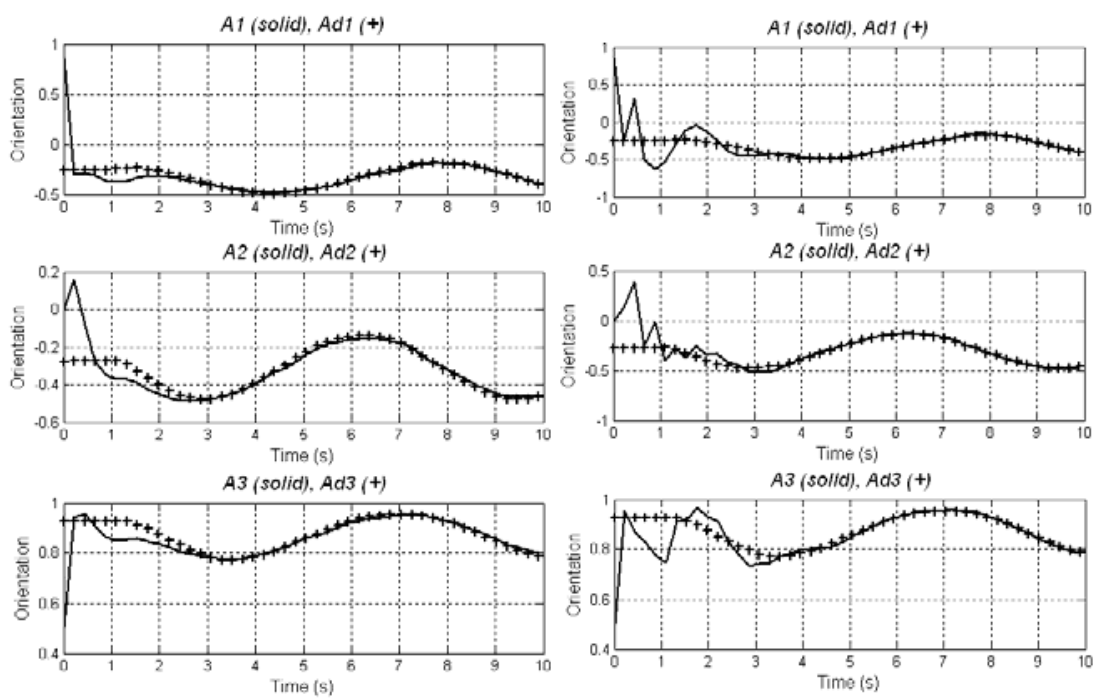

Fig. 3. Orientation Variables and References for DISMC (Left) and for PID controller (Right)
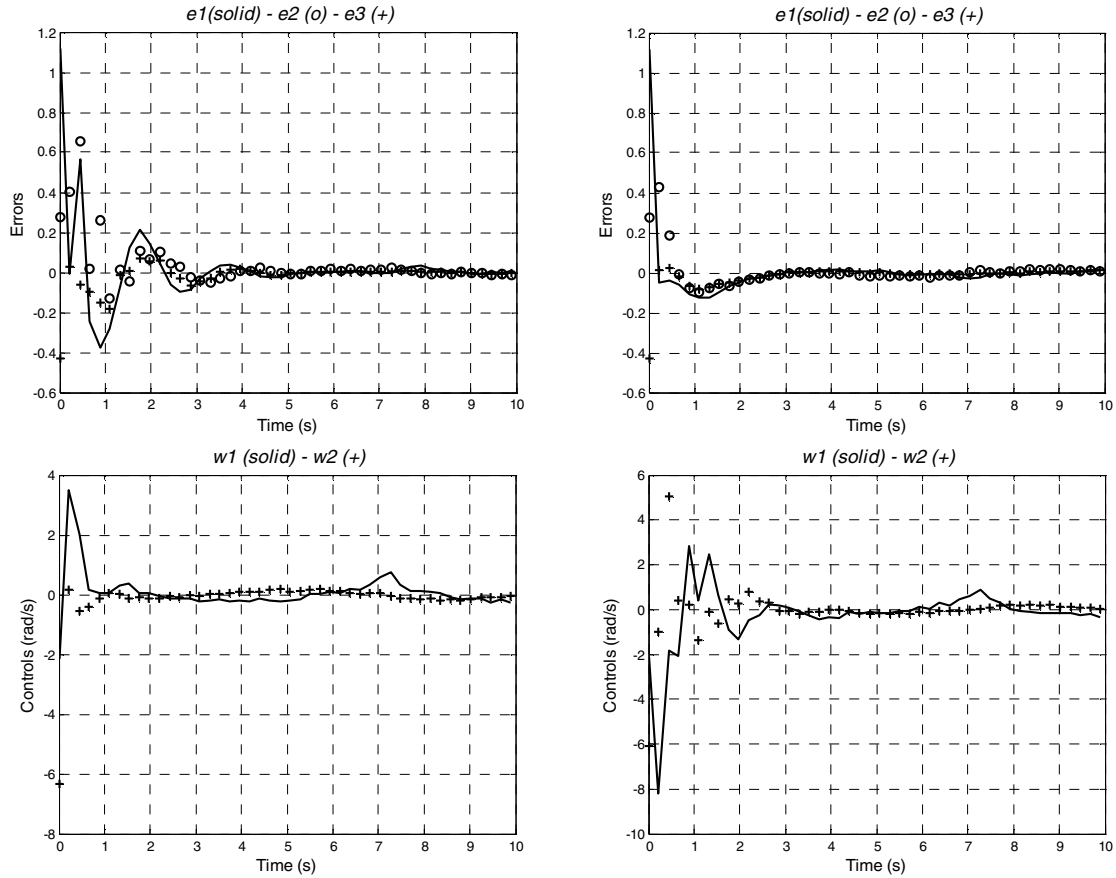

Fig. 4. Error variables in DISMC (Top Left). Error variables in PID controller (Top Right). Control signals in DISMC (Bottom Left). Control signals in PID controller (Bottom Right). 
PID, this can be observed in Figure 4. Also, a lower overshoot and a better transient response are noted in DISMC simulation. The angular velocities (control signals) of the joints of the PTU are shown in Figure 4 as well. Note the lower magnitude of the control signals in DISMC and the absence of high frequency components due to the use of a continuous control instead sign function, in comparison with standard Sliding Mode Control.

\section{Conclusions}

A Discrete Integral Sliding Mode (DISM) controller was designed for Visual Object Tracking and a kinematic model for a PTU was obtained using rotors in Geometric Algebra $G_{3,0,0}$, which is a simpler method than using matrices. The proposed algorithm demonstrates a satisfactory performance in output tracking problem, since it achieves a reduced steady state tracking error. A comparison with discrete PID controller was made and DISMC showed a better performance in aspects like softness in control signals, lower magnitude in transient response, settling time and overshoot. The procedure for obtain the DISM controller is simple, and it can be applied to any kind of serial kinematical structure, moreover, the use of a continuous control law allows to ensure chattering-free SM motion. So, it can be concluded that ISMC is a good approach to solve the Visual Object Tracking problem.

\section{References}

1. Kragic, D., Miller, A.T., Allen, P.K.: Real-time tracking meets online grasp planning. In: International Conference on Robotics and Automation (ICRA), Seoul, Republic of Korea, pp. 2460-2465 (2001)

2. Lowe, D.G.: Object recognition from local scale-invariant features. In: International Conference on Computer Vision (ICCV), Corfu, Greece, pp. 1150-1517 (1999)

3. Swain, M.J., Ballard, D.H.: Color Indexing. International Journal of Computer Vision 7, 11-32 (1991)

4. Craig, J.J.: Adaptive Control of Mechanical Manipulators. Ed.Addison-Wesley, Reading (1988)

5. Ozaki, T., Susuki, T., Furuhashi, T., Okuma, S., Uchikawa, Y.: Trajectory Control of robotic Manipulator Using neural Networks. IEEE Transactions on Industrial Electronics 39(6), 555-570 (1992)

6. Utkin, V.I., Guldner, J., Shi, J.: Sliding Mode Control in Electromechanical Systems. Ed. Taylor and Francis, UK (1999)

7. Utkin, V.I., Shi, J.: Integral sliding mode in systems operating under uncertainty. In: IEEE Conference on Decision and Control CDC 1996, Kobe, Japan (1996)

8. Angeles, J.: Fundamentals of Robotic Mechanical Systems: Theory, Methods, and Algorithms, 2nd edn. Ed. Springer, USA (2002)

9. Utkin, V.: Sliding mode control in discrete-time and difference systems. In: Zinober, A.S.I. (ed.) Variable Structure and Lyapunov Control, ch. 5, vol. 193, pp. 87-107. Springer, New York (1994)

10. Zamora, J., Bayro, E.: Kinematics and Differential Kinematics of Binocular Heads. In: Proc. of the Int. Conf. of Robotics and Automation ICRA 2006, Orlando Florida, USA, pp. 4130-4135 (2006) 